\title{
CARACTERIZAÇÃO FISIOLÓGICA DA REDUÇÃO DE CRESCIMENTO DE MUDAS DE CITROS MICORRIZADAS EM ALTAS DOSES DE FÓSFORO ${ }^{(1)}$
}

\author{
J. O. A. SENA ${ }^{(2)}$, C. A. LABATE ${ }^{(3)}$ \& E. J . B. N. CARDOSO ${ }^{(4)}$
}

\begin{abstract}
RESUMO
Com o objetivo de estudar a redução de crescimento de mudas de citros micorrizadas, instalou-se um experimento em casa de vegetação com seis doses de $P\left(0,50,100,150,200\right.$ e $\left.250 \mathrm{mg} \mathrm{kg}^{-1}\right)$, sem e com inoculação do Fungo Micorrízico Arbuscular (FMA) Glomus intraradices. Como porta-enxerto, usou-se tangerineira 'Cleópatra' (Citrus reshni). O substrato usado foi uma mistura de amostra de terra (Neossolo) e areia, na proporção de 3:1, com baixa concentração de $\mathbf{P}$ disponível. O delineamento estatístico foi inteiramente casualizado, disposto em um fatorial $6 \times 2$, com cinco repetições. Seis meses após o transplantio, as plantas foram avaliadas por meio das seguintes variáveis: altura de plantas, matéria seca da parte aérea, transpiração, resistência estomática e área foliar. Nas doses mais altas de $\mathbf{P}$, as plantas micorrizadas apresentaram redução na altura, na matéria seca da parte aérea e na área foliar, menor taxa de transpiração e maior resistência estomática, quando comparadas com o controle não micorrizado. Considerando estes resultados, a redução no crescimento de mudas micorrizadas em alta dose de $P$ pode estar relacionada, em parte, com a existência de forte dreno de carboidratos na raiz.
\end{abstract}

Termos de indexação: fungo micorrízico arbuscular, área foliar, transpiração, resistência estomática.

\footnotetext{
(1) Parte da Tese de Doutorado do primeiro autor. Projeto financiado pela FAPESP. Recebido para publicação em agosto de 2002 e aprovado em agosto de 2004.

(2) Professor do Departamento de Agronomia da Universidade Estadual de Maringá - UEM. Av. Colombo 5790, CEP 87020-900 Maringá (PR). E-mail: joasena@uem.br

(3) Professor do Departamento de Genética, Escola Superior de Agricultura "Luiz de Queiroz" - ESALQ/USP. Caixa Postal 09, CEP 13418-900 Piracicaba (SP). E-mail: calabate@esalq.usp.br

(4) Professor do Departamento de Solos e Nutrição de Plantas, ESALQ/USP. E-mail: ejbncard@esalq.usp.br
} 


\title{
SUMMARY: PHYSIOLOGICAL CHARACTERIZATION OF GROWTH DEPRESSION IN ARBUSCULAR MYCORRHIZAL CITRUS SEEDLINGS UNDER HIGH P LEVELS
}

\begin{abstract}
An experiment was installed under greenhouse conditions to study growth depression in mycorrhizal citrus rootstocks at six P levels $\left(0,50,100,150,200\right.$, and $\left.250 \mathrm{mg} \mathrm{kg}^{-1}\right)$, with and without (controls) inoculation of the arbuscular mycorrhizal fungus (AMF) (Glomus intraradices). The rootstock used was Citrus reshni Hort. Ex Tanaka, cv. Cleópatra, grown in an autoclaved, limed mixture (3:1) of a soil with low available $\mathrm{P}$ and washed sand. There were five replicates per treatment, in a random $6 \times 2$ factorial design. Six months after transplanting the seedlings, the plants were harvested and analyzed. The following characteristics were considered: plant growth, shoot dry matter, leaf area, transpiration and stomatal resistance. At thehighest P levels, mycorrhizal plants presented lower dry matter and plant height values, lower leaf transpiration, and higher stomatal resistance, when compared to non-mycorrhizal plants. Therefore, growth depression of mycorrhizal citrus plants at high P levels may be related to a strong carbon sink in mycorrhizal citrus roots.
\end{abstract}

Index terms: arbuscular mycorrhizal fungi, leaf area, transpiration, stomatal resistance.

\section{NTRODUÇÃO}

Algumas espécies de plantas, quando micorrizadas e cultivadas em doses altas de $\mathrm{P}$, apresentam redução no crescimento e, às vezes, na produção de massa seca da parte aérea. Esse fenômeno, conheci do como depressão de crescimento, pode também ocorrer em condições de campo. No caso de citros, a produção da cultura depende do preparo de mudas de alta qualidade, sendo, via de regra, usadas grandes quantidades de insumos, em especial os adubos. Nessas condições, a inoculação de fungos micorrízicos em porta-enxertos fica comprometida, uma vez que o benefício resultante dessa prática poderá deixar de existir ou afetar a produção de mudas.

Por essa razão, a compatibilização entre portaenxerto, a espécie de fungo mi corrízico arbuscular e a quantidade de insumos, especialmente os adubos fosfatados, é de fundamental importância para a produção racional de mudas de citros. A pesquisa tem demonstrado a importância estratégica dos fungos micorrízicos arbusculares para a produção de mudas e culturas em condições tropicais (Cardoso et al., 1986; Colozzi-Filho \& Siqueira, 1986), caracterizadas pelos solos ácidos e com alta capaci dade de fixação de $P$.

Em condições de baixos níveis de $\mathrm{P}$, benefícios, tais como: aumento na absorção de nutrientes (Lambert et al., 1979; Antunes \& Cardoso, 1991), em especial de $P$, redução de efeitos tóxicos de metais como manganês (Cardoso, 1996; Gomes, 1997), aumento das taxas de fotossíntese (Drüge \& Schönbeck, 1992; Sena, 1998), aumento deárea foliar e da biomassa vegetal, maior massa seca da parte aérea ealtura de plantas (Antunes \& Cardoso, 1991), além dos benefícios relacionados com a sanidade vegetal, têm sido verificados e qualificam a prática de inoculação com fungos mi corrízicos arbusculares como fundamental e indispensável à viabilização do processo produtivo nas nossas condições. A eficiência e a intensidade desses benefícios vão depender de fatores associados ao hospedeiro, ao microssimbionte e a condições edafocl imáticas.

Nesse contexto, a pesquisa tem avançado no sentido não só de estabel ecer o cultivo desses fungos em culturas monoxênicas com segmentos de raízes, mas também de verificar a viabilização de inóculo (Bécard \& Piché, 1989; Bécard et al., 1992), o que será também de importância vital para estudar, em simbiose e em separado, o hospedeiro e o fungo MA.

Um dos benefícios imediatos da introdução e do uso efetivo dessa prática na agricultura brasileira será a redução do uso de insumos e, em especial, de adubos, concorrendo para reduzir custos de produção, viabilizar a produção de grandes culturas e pequenos sistemas de produção, beneficiando o meio ambiente. Entender em níveis fisiológicos e bioquímicos a ocorrência de depressão de crescimento em plantas micorrizadas com fungos MA, quando cultivadas em doses altas de fosfato, é de fundamental importância para a adesão da prática e produção de mudas cítricas com altos padrões agronômicos (produção x qualidade x custo x meio ambiente).

Em termos fisiológicos e bioquímicos, algumas respostas das plantas aos fungos arbusculares têm sido verificadas, principalmenteem níveis baixos de $P$, tais como: aumento da atividade de ATPase na membrana periarbuscular, aumento da atividade de 
invertase, alteração do nível dehormônios, aumento da taxa de respiração das raízes, das taxas de fotossíntese, da absorção de nutrientes, da taxa de crescimento e de área foliar, aumento das taxas de transpiração e redução da resistência estomática; síntese de proteínas relacionadas com a simbiose (Smith \& Gianinazzi-Pearson, 1988; Kothari et al., 1990; Drüge \& Schönbeck, 1992; Danneberg et al., 1992; Rekoslavskaya \& Bandurski, 1994).

N este trabal ho, são discutidas al gumas características fisiológicas de porta-enxertos micorrizados, cultivados em diferentes doses de $\mathrm{P}$, com o objetivo de gerar informações que permitam entender a ocorrência de redução no crescimento de mudas em altos níveis de $P$.

\section{MATERIAL E MÉTODOS}

O experimento foi realizado em casa de vegetação no Departamento de Sol os e Nutrição de Plantas da ESALQ/USP. U sou-se, como substrato, uma mistura constituída de amostra de terra proveniente do horizonte A de Neossolo e de areia lavada, na proporção de 3:1, queapresentou, após autoclavagem, as seguintes características químicas: $\mathrm{pH}\left(\mathrm{CaCl}_{2}\right) 3,9$; MO $12 \mathrm{~g} \mathrm{~kg}^{-1} ; \mathrm{P}$ (Resina) $4 \mathrm{mg} \mathrm{dm}^{-3} ; \mathrm{K}, \mathrm{Ca}, \mathrm{Mg}, \mathrm{H}+\mathrm{Al}$, respectivamente, $0,06,0,2,0,2,3,4 \mathrm{cmol}_{c} \mathrm{dm}^{-3}$. O substrato, após autoclavagem, foi corrigido com cal cário dolomítico, visando aumentar a saturação por bases para $65 \%$ (4,5 g de cal cário por vaso com $5 \mathrm{~kg}$ desubstrato). Os nutrientes foram aplicados em solução, usando-se $\left(\mathrm{NH}_{4}\right)_{2} \mathrm{SO}_{4}\left(80 \mathrm{mg} \mathrm{kg}^{-1}\right.$, metade aos 30 emetade aos 60 dias do transplantio das mudas), $\mathrm{KCl}\left(50 \mathrm{mg} \mathrm{kg}^{-1}\right)$ e $1 \mathrm{~mL}$ por vaso de uma solução dos micronutrientes constituintes da solução de Sarruge (1975) menos o Mn e $1 \mathrm{~mL}$ de Fe-EDTA da solução de Hoagland \& Arnon (1950).

U sou-se, como porta-enxerto cítrico, a tangerineira 'Cleópatra' (Citrus reshni Hort. Ex Tanaka, cV Cleópatra) e, como doses de P: 0, 50, 100, 150, 200 e $250 \mathrm{mg} \mathrm{kg}^{-1}$ de substrato na forma de superfosfato triplo, com e sem fungo micorrízico arbuscular (Glomus intraradices) (FMA), aplicado na quantidade de 20 g de sol o rizosférico, obtido de vaso de multiplicação, onde se cultivou Brachiaria decumbens.

O experimento foi instalado de acordo com o delineamento experimental inteiramente casualizado, com cinco repetições, disposto em um fatorial $6 \times 2$ (seis doses de $P$ e dois níveis de micorrização, sem e com). Seis meses após o transplantio, as plantas foram colhidas e as seguintes características avaliadas:

1. Altura de plantas: medida a partir do colo da planta até o meristema apical.

2. Massa seca da parte aérea: fol has e caules foram colhidos, postos para secar em estufa com circulação forçada de ar a $65^{\circ} \mathrm{C}$, por $48 \mathrm{~h}$, e, em seguida, pesados em balança semi-analítica.

\section{Transpiração e resistência estomática:} medidas com porômetro model o LI-1600.

4. Área foliar: determinada a partir da coleta de discos fol iares, usando-se vasadores com diâmetro previamente conhecido. Os discos foliares col etados foram secos em estufa com circulação forçada de ar a $65^{\circ} \mathrm{C}$ por $48 \mathrm{~h}$. O cálculo de área foliar foi feito considerando a massa dos discos foliares e a massa de fol ha por planta.

5. Percentagem de colonização radicular interna: as raízes foram coletadas, cortadas e coradas pelo método de coloração e clarificação de Phillips \& Hayman (1970). A quantificação da colonização foi feita pelo método de Giovannetti \& Mosse (1980) e Bethlenfalvay et al. (1981).

\section{RESULTADOS E DISCUSSÃO}

Em baixas doses de fosfato, as plantas micorrizadas apresentaram maior altura, quando comparadas com as plantas-controle(Figura la). Na dose mais alta de $\mathrm{P}, 250 \mathrm{mg} \mathrm{kg}^{-1}$, percebeu-se uma tendência de redução da altura das plantas inoculadas com FMA. Tendência semelhante ocorreu com a produção de matéria seca da parte aérea (Figura 1b). Este efeito de benefício em favor das plantas micorrizadas em baixas doses de $\mathrm{P}$, bem como a tendência de decréscimo com o aumento da dose do elemento, pôde ser verificado igualmente quando se observou a área fol iar (F igura 1c). Efeitos semel hantes têm sido observados por outros autores (Peng et al., 1993; Gomes, 1997).

Com base nas curvas de respostas das variáveis anal isadas e nas equações de regressão geradas em cada modelo, pôde-se calcular a dose de $P$ no substrato a partir da qual se estabel eceu a transição entre a simbiose mutualística e o parasistismo, evidenciado pela redução de crescimento, redução da produção de matéria seca e área foliar (Figuras la, b e c). Para o caso em questão, a dose calculada correspondeu a $240 \mathrm{mg}^{\mathrm{de}} \mathrm{P} \mathrm{kg}^{-1}$ de substrato.

Em termos de transpiração e resistência estomática, destacam-se dois pontos da curva: zero e $250 \mathrm{mg} \mathrm{kg}^{-1}$ de $\mathrm{P}$ de substrato (Figura 2), como sendo de importância chave para entender o sistema estudado e estabel ecer correlação dessas variáveis com as respostas na altura de plantas, produção de matéria seca da parte aérea e área foliar. $\mathrm{Na}$ condição limitante de $\mathrm{P}$ disponível no solo $\left(0 \mathrm{mg} \mathrm{kg}^{-1}\right.$ de P) (Figura 2), o sistema micorrizado tendeu a apresentar níveis mais reduzidos de transpiração e mais elevados de resistência estomática, quando 


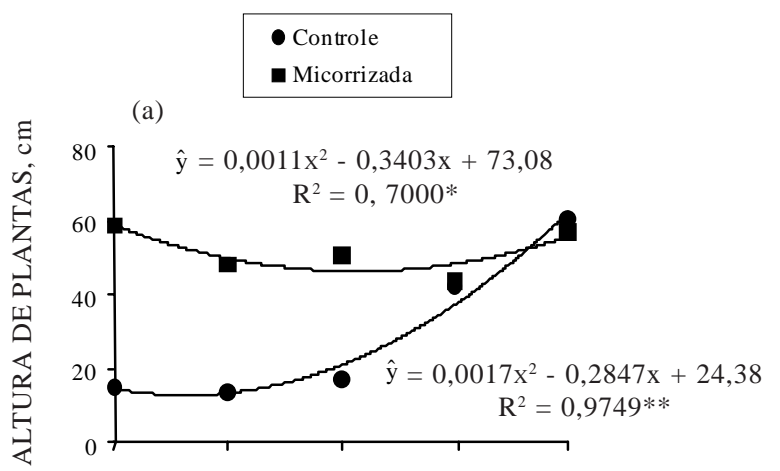

(b)

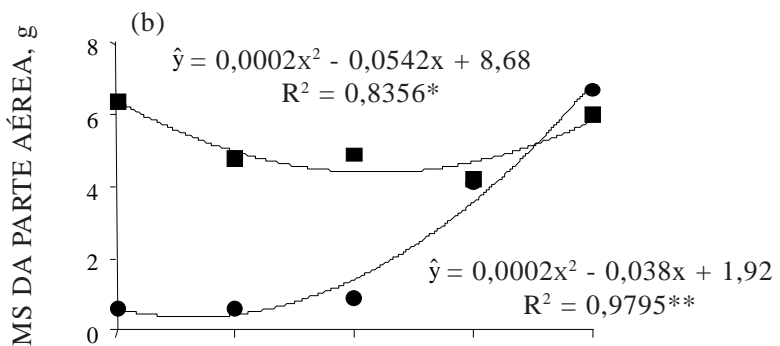

(c)

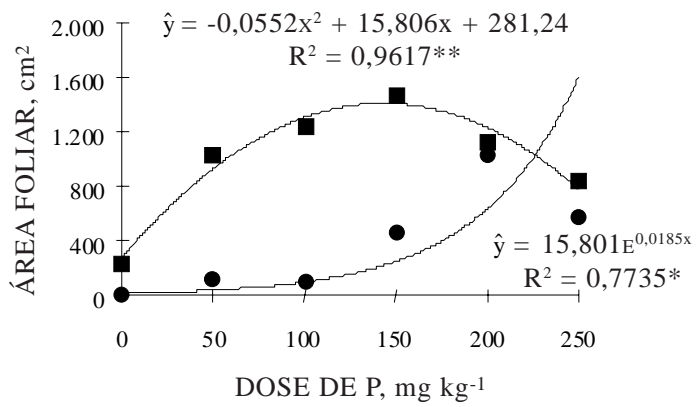

Figura 1. Altura de plantas (a), matéria seca da parte aérea (MS) (b) e área foliar (c) da tangerineira "Cleópata" inoculadas ou não com fungo micorrízico arbuscular e cultivadas com diferentes doses de $\mathbf{P}$.

comparados com os do sistema não inoculado com FMA. A mesma tendência pôde ser observada no extremo oposto, em dose alta de P $\left(250 \mathrm{mg} \mathrm{kg}^{-1}\right)$. É importante ressaltar que a col onização radicular interna tendia a decrescer com o aumento das doses de $P$, atingindo níveis bastante reduzidos, próximos a zero, na máxima dose de $P$ (colonização radicular interna: $0=27,8 ; 50=20,4 ; 100=14,7 ; 150=4,6$; $200=0,1 ; 250=1,2 \%$ ) (Melloni \& Cardoso, 1999).

Em dose baixa de $P$, as plantas micorrizadas apresentaram, como visto, redução das taxas de transpiração e aumento da resistência estomática (Figura 2), apesar de, classicamente, plantas micorrizadas em baixo nível de fosfato tenderem a apresentar o contrário (Smith, 1980; Kothari et al., 1990). No entanto, a redução detaxa detranspiração tem sido verificada (Drüge \& Schönbeck, 1992). Estas reduções podem ocasionar fechamento de estômatos ou restrição à difusão de gases, como de $\mathrm{O}_{2}$ e $\mathrm{CO}_{2}$.
Como, nesta condição, as plantas micorrizadas apresentaram maiores val ores de al tura e produção de matéria seca, infere-se que, para haver este ganho em termos de biomassa, deve ter havido aumento nas taxas de fotossíntese, ou seja, aumento na assimilação do $\mathrm{CO}_{2}$, o que poderia ser atribuído à presença benéfica do FMA nosistema, por favorecer aumento na absorção de micro e macronutrientes, em especial, nesse caso, de $\mathrm{P}$, elemento-chave na produção de fotossintatos.

Os possíveis aumentos nas taxas de fotossíntese podem ser respaldados pelo aumento marcante na área foliar das plantas micorrizadas até a dose de $150 \mathrm{mg} \mathrm{kg}^{-1}$ de $\mathrm{P}$ de substrato. Assim, é possível que a energia que poderia ser perdida no processo respiratório esteja sendo canal izada para a produção de carboidratos, em especial sacarose, para acúmulo de biomassa vegetal e, conseqüentemente, crescimento. Por outrolado, na mais alta dose deP, a ocorrência de redução das taxas de transpiração, aumento da resistência estomática e tendência de redução na produção de biomassa vegetal e área foliar poderia ser explicada pela redução da colonização interna e distribuição de estruturas extra-radiculares do fungo ativas no sistema (Melloni \& Cardoso, 1999). Uma vez existindo mi célio extra-radicular ativo no sistema micorrizado, pode-se estabelecer um dreno de carboidratos nas raízes do hospedei ro que, nesta condição, não contará com os retornos por parte do fungo, o qual necessitaria estar completamente estabelecido no interior das células do hospedeiro. Esse dreno de sacarose, não sendo compensado por aumentos em assimilação de $\mathrm{CO}_{2}$ (aumento na resistência estomática erestrição a trocas gasosas), resultaria em queda de produção de bi omassa, altura ematéria seca.

Deve-se estabelecer aqui que as reduções na colonização interna poderiam talvez ser explicadas pela alta concentração de $P$ nas células das raízes micorrizadas, em relação ao meio externo, o que demandaria energia a ser despendida pelo hospedei ro para absorver o el emento (gradiente de potencial el etroquímico). Nessa condição, o hospedeiro passaria a limitar a existência de estruturas internas do fungo, consideradas de alto custo energético de manutenção (Graham \& Eissenstat, 1994), e o sistema, no entanto, ajustarse-ia à nova situação, num novo patamar. Ocorreria, portanto, um ajuste da interação à condição estabelecida, regulada pelo hospedeiro, provavelmente pel oteor de carboidratos, em especial sacarose e glicose (Graham et al., 1997).

Outra hipótese para explicar a redução de crescimento e produção de biomassa pode estar relacionada com um possível desbalanço hormonal, resultante de desequilíbrio nutricional. Neste caso, o sistema micorrizado, por absorver mais $\mathrm{P}$, favoreceria o acúmulo de fosfato inorgânico (polifosfatos) nas raízes do hospedeiro, resultando em interações com metais, como $\mathrm{Fe}, \mathrm{Mn}, \mathrm{Cu}$, e, 

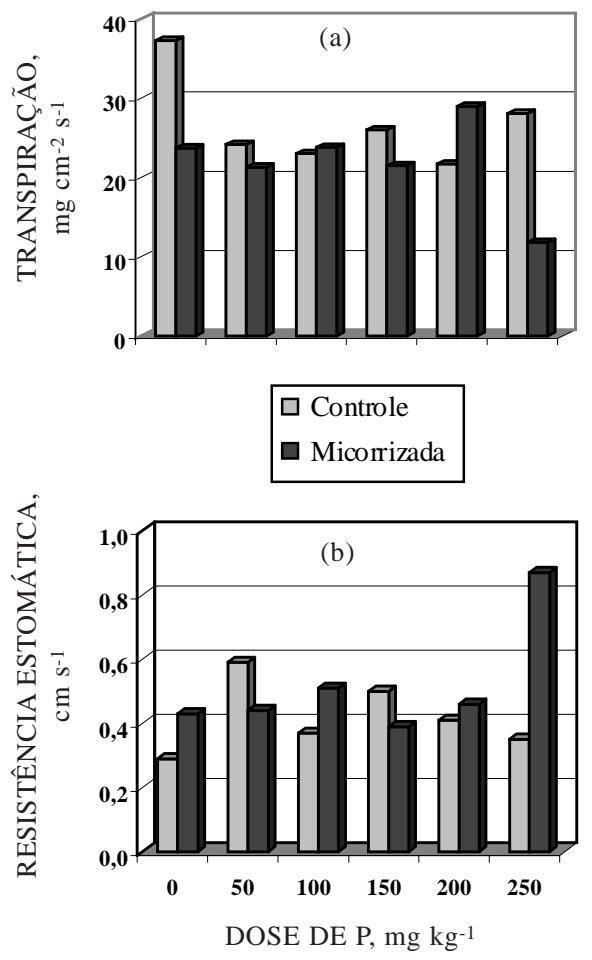

Figura 2. Transpiração (a) e resistência estomática (b) de plantas da tangerineira "Cleópata" inoculadas ou não com fungo micorrízico arbuscular e cultivadas com diferentes doses de $P$.

especialmente, $\mathrm{Zn}$. Isto restringiria o transporte de Zn para a parte aérea (Lambert et al., 1979; Cardoso, 1996), provocando redução na síntese de auxinas (alteração dos níveis de indol e serina, precursores de triptofano, aminoácido que origina o ácido indolacético) (Rekoslavskaya \& Bandurski, 1994), desbalanço da relação auxina/citocininas (redução de al ongamento celular, encurtamento de entrenós) e, finalmente, redução de crescimento. A intensidadade desse efeito dependeria, dentre outros fatores, da espécie ou mesmo variedade de planta. Outro hormônio que pode estar envolvido é o ácido abscísico (ABA) que, produzido na raiz, teria influência na abertura e fechamento de estômatos, controlando a transpiração e trocas gasosas. Tem sido verificado que raízes de plantas micorrizadas apresentam maiores concentrações desse inibidor de crescimento (Danneberg et al., 1992).

\section{CONCLUSÕES}

1. A inoculação de plantas cítricas com FMA alterou suas características fisiológicas.

2. Altas doses de $P$ reduziram o crescimento, a transpiração, a resistência estomática, a área foliar e a matéria seca da parte aérea do porta-enxerto de citros (tangerineira "Cleópatra").
3. O limite entre simbiose mutualística e parasitismo ficou evidenciado, para as variáveis avaliadas, em dose de $\mathrm{P}$ no substrato superior a $240 \mathrm{mg} \mathrm{kg}^{-1}$.

\section{LITERATURA CITADA}

ANTUNES, V. \& CARDOSO, E.J.B.N. Growth and nutrient status of citrus plants as influenced by mycorrhiza and phosphorus application. Plant Soil, 131:11-19, 1991.

BÉCARD, G. \& PICHÉ, Y. New aspects on the acquisition of biotrophic status by a vesicular-arbuscular mycorrhizal fungus, Gigaspora margarita. New Phytol., 1112:77-83, 1989.

BÉCARD, G.; DOUDS, D.D. \& PFEFFER, P.E. Extensive in vitro hyphal growth of vesicular-arbuscular mycorrhizal fungi in the presence of $\mathrm{CO}_{2}$ and flavonols. Appl. Environ. Microbiol., 58:821-825, 1992.

BETHLENFALVAY, G.J .; PACOVSKY, R.S. \& BROWN, M.S. Measurement of mycorrhizal infection in soybeans. Soil Sci. Soc. Am. J ., 45:871-875, 1981

CARDOSO, E.J.B.N.; ANTUNES, V.; SILVEIRA, A.P.D.; \& OLIVEIRA, M.H.A. Eficiência de fungos micorrízicos vesículo-arbusculares em porta-enxertos de citros. R. Bras. Ci. Solo, 10:25-30, 1986.

CARDOSO, E.J .B.N. Interaction of mycorrhiza, phosphate and manganese in soybean. In: EUROPEAN SYMPOSIUM ON MYCORRHIAS, 4., Granada, 1994. Proceedings. Granada, European Comission, Directorate-General XII, 1996. p.304306.

COLOZZI-FILHO, A. \& SIQUEIRA, J.O. Micorrizas vesículoarbusculares em mudas de cafeeiro. I. Efeito de Gigaspora margarita e adubação fosfatada no crescimento e nutrição. R. Bras. Ci. Solo, 10:199-206, 1986.

DANNEBERG, G.; LATUS, C.; ZIMMER, W.; HUNDESHAGEN, B.; SCHNEIDER-POETSCH, H.J \& \& BOTHE, H. Influence of Vesicular-Arbuscular Mycorrhiza on phytohormone balances in maize (Zea mays L.). J . Plant Physiol., 141:3339, 1992.

DRÜGE, U.\& SCHÖNBECK, F. Effect of Vesicular-Arbuscular Mycorrhizal infection on transpiration, photosynthesis and growth of Flax (Linum usitatissimum L.) in relation to cytokinin levels. J. Plant Physiol., 141:40-42, 1992.

GIOVANETTI, M. \& MOSSE, B. An evaluation of techniques for measuring vesicular arbuscular mycorrhizal infection in roots. New Phytol., 84:489-500, 1980.

GOMES, V.F.F. Níveis de fósforo e fungos micorrízicos arbusculares no desenvolvimento de porta-enxertos cítricos. Piracicaba, Escola Superior de Agricultura "Luiz de Queiroz", 1997. (Tese de Doutorado)

GRAHAM, J.H. \& EISSENSTAT, D.M. Host genotype and the formation and function of VA mycorrhizae. Plant Soil, 159:179-185, 1994.

GRAHAM, J.H.; DUNCAN, L.W. \& EISSENSTAT, D.M. Carbohydrate allocation patterns in citrus genotypes as affected by phosphorus nutrition, mycorrhizal colonization and mycorrhizal dependency. New Phytol., 135:335-343, 1997. 
HOAGLAND, D.R. \& ARNON, D.I. The water culture method of growing plants without soil. Berkeley, University of California, 1950. 32p.

KOTHARI, S.K.; MARSCHNER, H. \& GEORGE, E. Effect of VA mycorrhizal fungi and rhizosphere microorganisms on root and shoot morphology, growth and water relations in maize. New Phytol., 116:303-311, 1990.

LAMBERT, D.H.; BAKER, D.E. \& COLE J r., H. The role of Mycorrhizae in the interactions of phosphorus with zinc, copper, and other elements. Soil Sci. Soc. Am. J ., 43:976980, 1979.

MELLONI, R. \& CARDOSO, E.J.B.N. Quantificação de micélio extrarradicular de fungos micorrízicos arbusculares em plantas cítricas. II. Comparação entre diferentes espécies cítricas e endófitos. R. Bras. Ci. Solo, 23:59-67, 1999.

PENG, S.; EISSENSTAT, D.M.; GRAHAM, J .H.; WILLIAMS, K. \& HODGE, N.C. Growth depression in mycorrhizal citrus at high phosphorus supply: analysis of carbon costs. Plant Physiol., 101:1063-1071, 1993.
PHILLIPS, J.M. \& HAYMAN, D.S. I mproved procedures for clearing roots and staining parasitic and vesiculararbuscular mycorrhizal fungi for rapid assesment of infection. Trans. British Mycol. Soc., 55:158-160, 1970.

REKOSLAVSKAYA, N.I. \& BANDURSKI, R.S. Indole as a precursor of indole-3-acetic acid in Zea mays. Phytochemistry, 35:905-909, 1994.

SARRUGE, J .R. Soluções nutritivas. Summa Phytopathol., 1:231-233, 1975.

SENA, J .O.A. Caracterização bioquímica da depressão de crescimento de porta-enxertos cítricos inoculados com fungo micorrízico arbuscular em doses altas de fosfato. Piracicaba, Escola Superior de Agricultura "Luiz de Queiroz", 1998. (Tese de Doutorado)

SMITH, S.E. Mycorrhizas of autotrophic higher plants. Biol. Rev., 55:475-510, 1980.

SMITH, S.E. \& GIANINAZZI-PEARSON, V. Physiological interactions between symbionts in Vesicular-Arbuscular Mycorrhizal plants. Ann. Rev. Physiol. Plant Mol. Biol., 39:221-244, 1988. 